\title{
Summary of results on exotic searches from ATLAS
}

\author{
Jason Tam ${ }^{1, a}$, on behalf of the ATLAS Collaboration \\ ${ }^{1}$ Julius-Maximilians Universität Würzburg
}

\begin{abstract}
Despite the success of the Standard Model (SM) of particle physics in the recent decades describing nature within its capabilities, there remain open questions such as the naturalness of Electroweak Symmetry Breaking (EWSB) as well as potential deeper underlying symmetries of the SM that may contribute to the possibility of unifying the fundamental interactions. Supersymmetry (SUSY) is a very popular concept that is proposed to provide explanations to these issues, but there are also many other Beyond the Standard Model (BSM) concepts proposed that attempt to solve these remaining conundrums. The ATLAS experiment at the Large Hadron Collider (LHC) is designed to search for those non-SUSY BSM theories, referred to as Exotics. Presented here is a summary of selected results from different searches performed using proton-proton collisions at $\sqrt{s}=8 \mathrm{TeV}$ during 2012. As no significant excess were observed over SM background processes, the obtained results are interpreted as constraints on the possibility of the existence of these new physics.
\end{abstract}

\section{Introduction}

The LHC [1] has provided proton beams for collisions with a center of mass energy $\sqrt{s}=8 \mathrm{TeV}$ in the year 2012. The ATLAS [2] Experiment has recorded collision data totalling an integrated luminosity of $\mathscr{L}_{\text {int }}=20.3 \mathrm{fb}^{-1}$ that is suitable for physics analysis. This has led to the discovery of a new boson [3], which was later confirmed to be consistent with the predictions of SM Higgs boson [4, 5]. ATLAS is also sensitive to many potential signals of new physics, that are proposed as explanations for open questions of the SM, such as possible deeper underlying symmetries and the naturalness of EWSB. Many of these proposals may appear as distinct signatures at ATLAS, and searching for deviations from well-understood SM processes is an effective way to search for such signals. Exotic Searches at ATLAS can be seen as a collection of possible final states that can be used to search for new physics.

\section{Diboson Resonances}

\section{$2.1 \mathrm{WZ} / \mathrm{ZZ} \rightarrow l l q q$}

Narrow diboson resonances in the semi-leptonic decay channel $W Z / Z Z \rightarrow l l q q$ are predicted in many BSM models such as Technicolor [6-8], Warped Extra Dimensions [9-11] and Grand Unified Theories [12]. Two models are used as benchmarks for analysis optimization in the search for

\footnotetext{
ae-mail: jason.tam@cern.ch
} 
such resonances with $20 \mathrm{fb}^{-1}$ of data from $p p$ collisions at ATLAS: The Spin-2 Kaluza-Klein (KK) gravitons $\left(G^{*} \rightarrow Z Z\right)$ from the Extended Randall-Sundrum (RS) Model [13] of warped extra dimensions, and spin-1 $W^{\prime}$ gauge bosons $\left(W^{\prime} \rightarrow W Z\right.$ ) from the Extended Gauge Model (EGM) [14]. The Extended RS model allows the SM fields to propogate into the bulk of the extra dimension, and avoids constraints that are present in the original RS model such as limits on Flavour-ChangingNeutral-Currents (FCNC) and electroweak precision tests. It allows a dimensionless coupling constant $k / \bar{M}_{P l} \sim 1$, where $k$ is the curvature of the warped extra dimension, and $\bar{M}_{P l}=M_{P l} / \sqrt{8 \pi}$ is the reduced Planck mass. The Extended Gauge Model introduces $W^{\prime}$ and $Z^{\prime}$ bosons that have SM couplings to fermions. The coupling strength of $W^{\prime}$ to $W Z$ relative to the SM couplings is modified by a mixing factor $\xi=c \times\left(m_{W} / m_{W^{\prime}}\right)$, where $m_{W}$ and $m_{W^{\prime}}$ are respectively the masses for $W$ and $W^{\prime}$, and $c$ is a coupling scale factor.

The selection criteria for $W Z / Z Z \rightarrow l l q q$ events are defined independently for the electron and muon channels as appropriate, using optimal lepton selection criteria, which are independently studied. Jets are reconstructed from calorimeter clusters using the anti-kt algorithm $[15,16]$ with a distance parameter $R=0.4$. For resonances with a mass greater than $900 \mathrm{GeV}$, the two jets formed by the $q \bar{q}$ pair are often merged into a single jet, and the fraction of $q \bar{q}$ pairs merging increases with the resonance mass. These merged jets are labelled as large-R jets or " $J$ ", and are reconstructed with the Cambridge-Aachen Algorithm [17, 18] with a distance parameter of $R=1.2$. Regions that are sensitive to such resonances are divided into distinct kinematic regions based on the dilepton and hadronic system, namely the "low/high- $p_{T}$ resolved region" (LR/HR) and the merged region (MR). The mass spectrum of the $l l q q$ final state, is reconstructed with the mass of the dilepton and dijet $\left(m_{l l j j}\right)$ system in $\mathrm{LR} / \mathrm{HR}$, and with the mass of the dilepton and the large-R jet $\left(m_{l l J}\right)$ system in MR, as shown in Figure 1a. The results of both lepton channels are combined in these distributions to search for an excess with respect to the SM predictions. No significant excess is observed, and the results are intepreted as $95 \%$ CL limits for mass of $G^{*}$ with $k / \bar{M}_{P l}=1$ and 0.5 , as well as the mass of $W^{\prime}$.

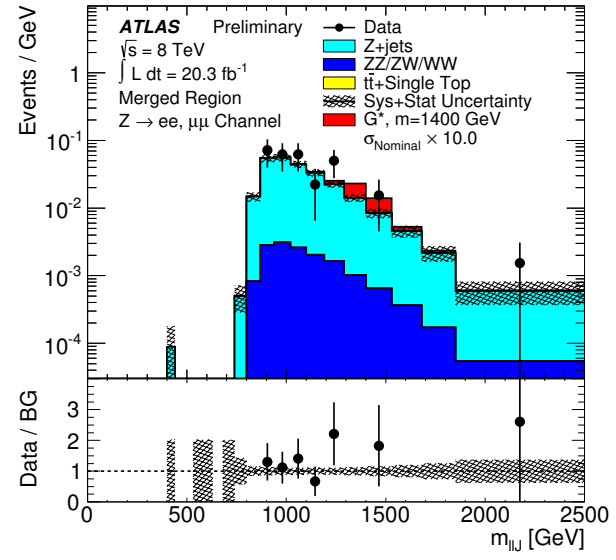

(a)

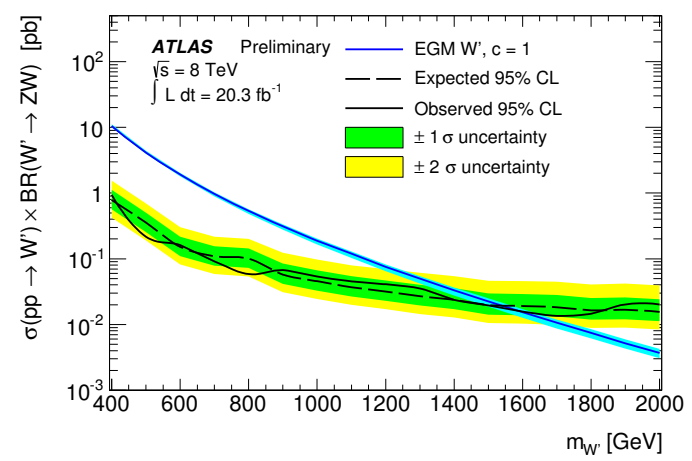

(b)

Figure 1: Shown in Figure 1a is the $m_{l l J}$ distribution in MR, and in Figure $1 \mathrm{~b}$ is the $95 \%$ CL limits on cross section times the branching ratio of the $W^{\prime} \rightarrow W Z$ process, as a function of $m_{W^{\prime}}$. [19] 


\section{$2.2 W Z \rightarrow l v l l$}

The search for diboson resonances at high masses in the leptonic decay channel $W Z \rightarrow l v l l$ uses the EGM model [12] that involves a spin-1 $W^{\prime}$ boson, and the heavy vector triplet (HVT) models described

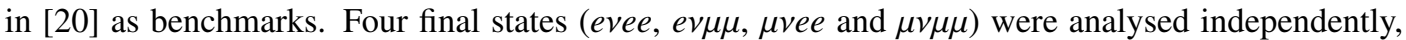
and then combined into the final result. The transverse momentum of the neutrino is estimated by the measurement of missing transverse momentum $E_{T}^{\text {miss }}$. The reconstructed mass of the $W Z \rightarrow l v l l$ system is then used as the discriminating variable to search for an excess over SM expectations, and are divided into a low mass (LM, $m_{W^{\prime}} \lesssim 250 \mathrm{GeV}$ ) and a high mass (HM, $m_{W^{\prime}} \gtrsim 250 \mathrm{GeV}$ ) region. Figure 2a shows the reconstructed mass distribution in the HM signal region. The contributions from all four final states are combined in these two signal regions. A $p$-value of 0.08 at $375 \mathrm{GeV}$ (equivalent to a $1.75 \sigma$ local excess) has been observed. As this excess is not significant, the results are interpreted as 95\% CL limits on the mass of new heavy bosons, as shown in Figure $2 \mathrm{~b}$.

The couplings of the new fields to the fermions and gauge bosons as predicted by the HVT models are defined in terms of parameters in the generic Lagrangian. The triplet field couples to the fermionic current through the combination of $g^{2} c_{F} / g_{V}$ and to the Higgs and vector bosons through $g_{V} / c_{H}$. The parameter $g$ denotes the $S U(2)_{L}$ gauge coupling, $g_{V}$ is the coupling strength to vector bosons, and the presence of $c_{F}$ and $c_{H}$ are to allow the modification of couplings. Exclusion contours for resonance masses of $1,1.5$ and $2 \mathrm{TeV}$ are also obtained in the HVT parameter space of $\left[g^{2} c_{F} / g_{V}, g_{V} / c_{H}\right]$.
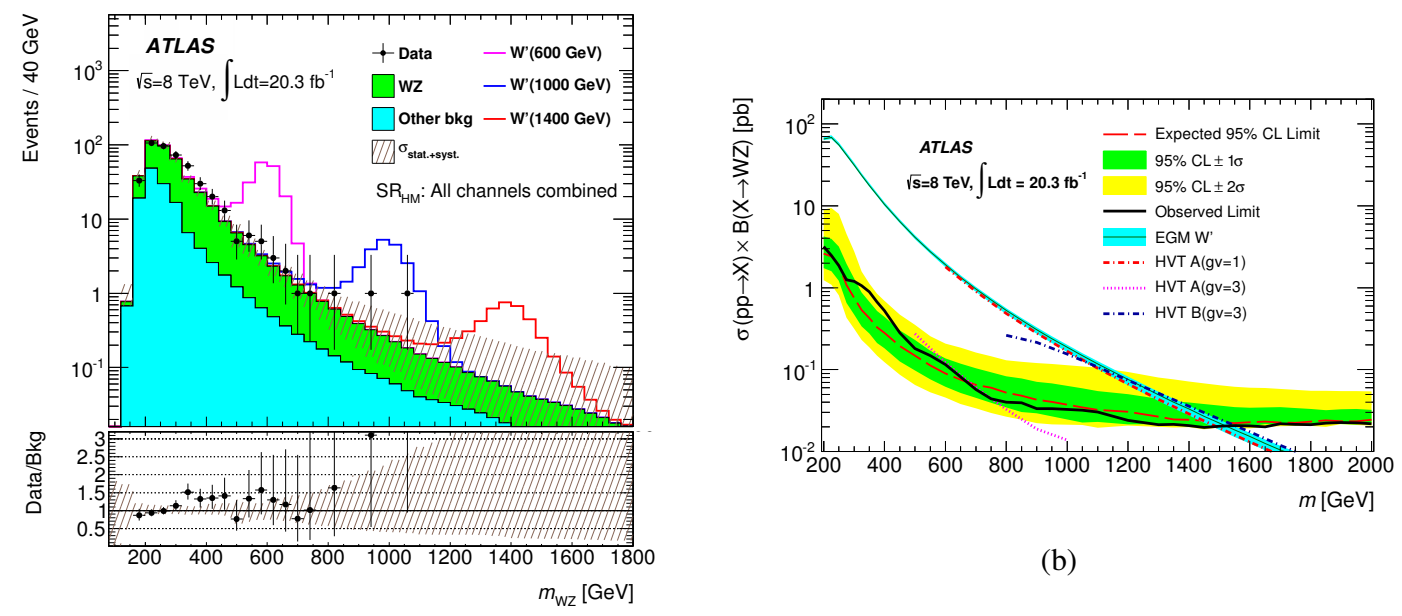

(b)

(a)

Figure 2: From [21], shown in Figure 2a is the distribution of the reconstructed mass $m_{W Z}$ in the $W Z$ system, and in Figure $2 \mathrm{~b}$ is the $95 \%$ CL limits on cross section of the resonance times the branching ratio of the resonance to the $W Z$ system, as a function of signal mass $m$.

\section{Dijet Resonances}

New particles or excitations in physics models can appear as $s$-channels resonances that have a sizable branching ratio to final states with quarks and gluons. Such resonances can appear as "bumps" in the 
dijet invariant mass $\left(m_{j j}\right)$ spectrum if they are sufficiently narrow. Considered benchmark models include two forms of chiral $W^{*}$ bosons [23-26] and Quantum Black Holes (QBH) [27-30] decaying into a mixture of quarks and gluons, excited quarks $\left(q^{*}\right)[31,32]$ decaying to $q g$, colour octet scalars (s8) [33-36] decaying to $g g$, and heavy $W^{\prime}[12,14,37-42]$ decaying to $q \bar{q}$.

A data-driven background estimate, derived from fitting a smooth function over observed data, is used in the search for an excess over SM expectations in the $m_{j j}$ spectrum. ATLAS and other experiments have shown in previous studies that the function $f(x)=p_{1}(1-x)^{p_{2}} x^{p_{3}+p_{4} \ln x}$ provides a satisfactory fit to the QCD prediction for the dijet production, where $p_{i}$ are fit parameters and $x=$ $m_{j j} / \sqrt{s}$. As shown in Figure 3 a, this function allows for smooth background variations, but does not accomodate localised excesses that may indicate the presence of new physics. With no presence of an significant excess observed over the SM expectations, the final results are interpreted as 95\% CL limits on the cross section times acceptance $(\sigma \times A)$ as a function of mass of the new physics $m_{N P}$ for the various benchmark models, as shown in Figure $3 \mathrm{~b}$ for the excited quarks.

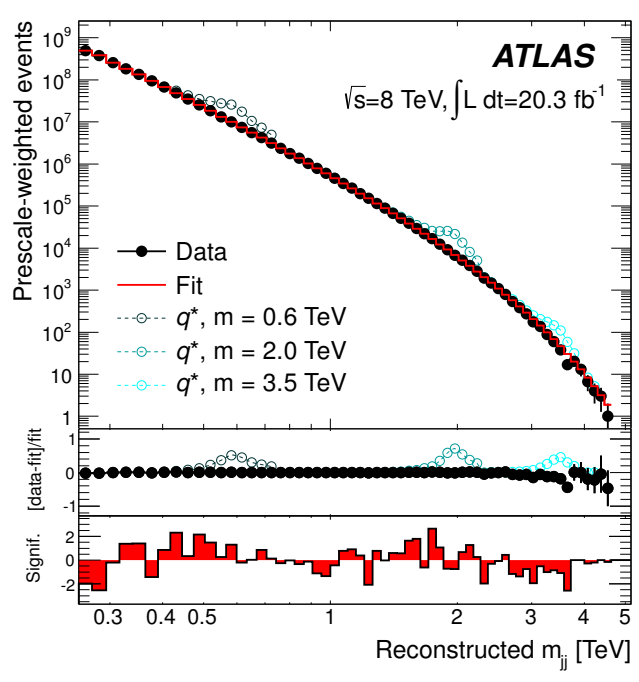

(a)

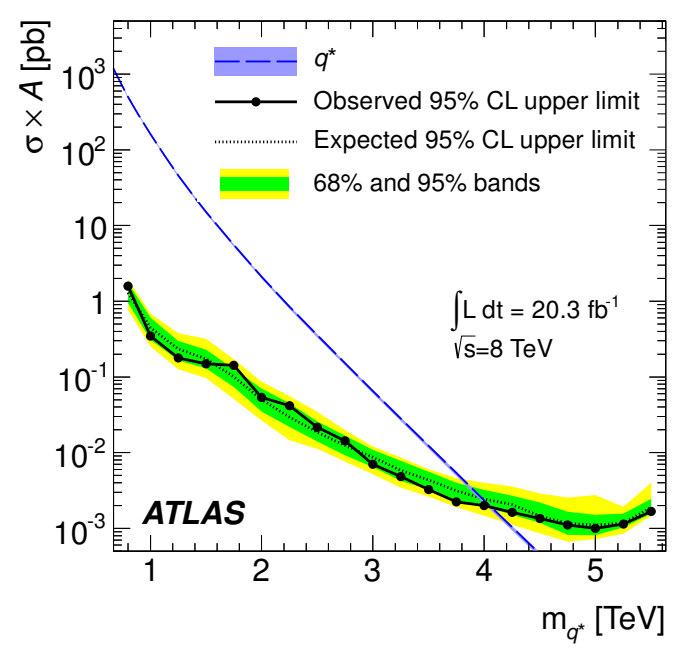

(b)

Figure 3: Shown in Figure 3a is the $m_{j j}$ distribution used to search for resonances, and in Figure $3 \mathrm{~b}$ is the $95 \%$ CL limits on cross section times the acceptance as a function of $m_{q^{*}}$. [22]

\section{Searches for $W^{\prime} \rightarrow t b$ decays}

Highly boosted top and bottom quarks detected in ATLAS can appear as decay products of a heavy $W^{\prime}$ boson. A search for its presence was performed with an analysis on the $W^{\prime} \rightarrow t \bar{b} \rightarrow W b \bar{b} \rightarrow q \bar{q} b \bar{b}$ decay mode for masses of $W^{\prime}$ above $1.5 \mathrm{TeV}$. With this final state it is possible to explore models that are potentially inaccessible to $W^{\prime} \rightarrow l v$. Such heavy $W^{\prime}$ bosons can appear in BSM models such as Technicolour [43], Little Higgs [44] and models involving extra dimensions such as Kaluza-Klein excitations of the SM W boson [45]. An effective model [46] with description of couplings to fermions is used as a benchmark in the analysis. It is assumed that the there are no light right-handed neutrinos 
that the $W^{\prime}$ boson would decay into, allowing only fully-hadronic decays in the right-handed sector. Both right-handed $\left(W_{R}^{\prime}\right)$ and left-handed $\left(W_{L}^{\prime}\right)$ models for the heavy bosons are used to generate the Monte-Carlo (MC) signal samples at different $m_{W^{\prime}}$. Coupling strengths to quarks for the $W^{\prime}$ bosons are assumed in the model to be the same as the SM W boson: $g_{R}=g_{S M}$ and $g_{L}=0$ for the $W_{R}^{\prime}$ boson, and $g_{R}=0$ and $g_{L}=g_{S M}$ for the $W_{L}^{\prime}$ boson, where $g_{S M}$ is the SM $S U(2)$ coupling strength. Events are analysed independently in one $b$-tag and two $b$-tag categories, and the results are independently combined for the $W_{L}^{\prime}$ and $W_{R}^{\prime}$ bosons. A data-driven background estimate is used to obtain a fit function that describes the background contributions from Multijet events, which is estimated to be $99(88) \%$ of the total background events in the one(two) $b$-tag category. Figure 4a shows the fit to data as well as the comparison to $W_{L}^{\prime}$ signal at different mass values. As no significant excess of events were observed for both $W_{L}^{\prime}$ and $W_{R}^{\prime}$, the final results are independently intepreted as 95\% CL Limits on the cross section times the branching ratio as a function of the $W^{\prime}$ mass, as shown in Figure $4 \mathrm{~b}$ for $W_{L}^{\prime}$. Limits for the coupling ratios as a function of the $W^{\prime}$ mass were also obtained at $95 \% \mathrm{CL}$, as shown in Figure $4 \mathrm{c}$ for $W_{R}^{\prime}$.

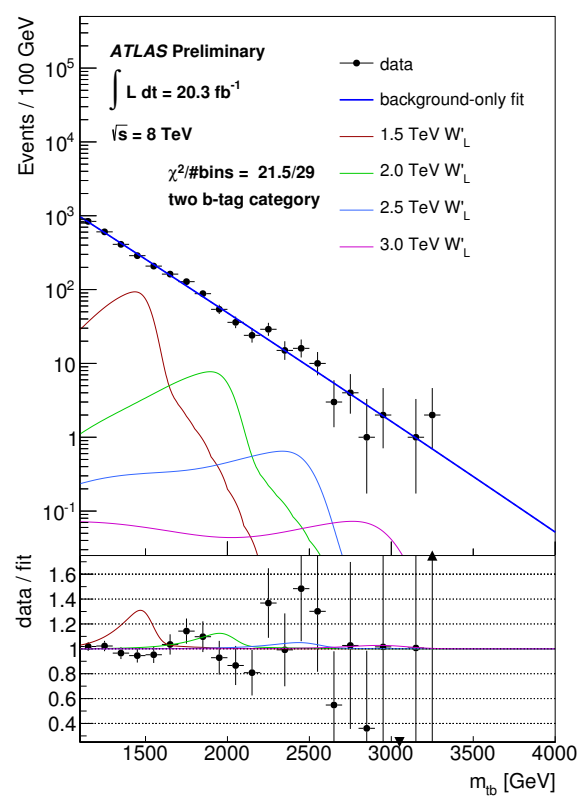

(a)

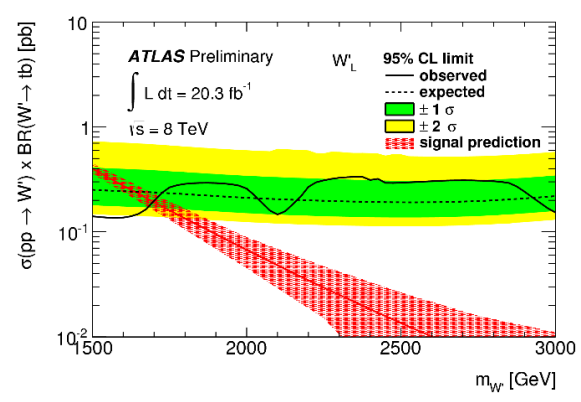

(b)

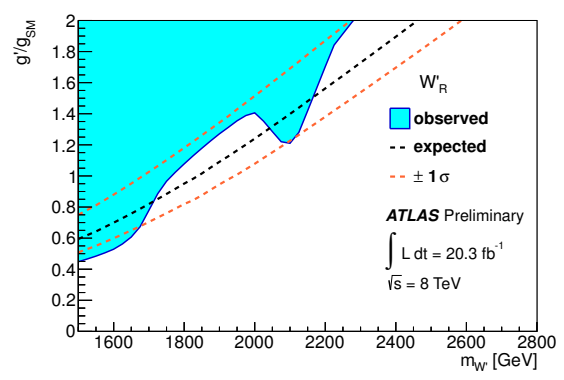

(c)

Figure 4: From [47], Figure 4a shows the comparison of the data to the fitted background and potential signals of new physics. Figure $4 \mathrm{~b}$ displays the $95 \%$ CL limits on cross section times the branching ratio as a function of $m_{W_{L}^{\prime}}$, and in Figure $4 \mathrm{c}$ is the $95 \%$ CL limits on coupling ratio as a function of $m_{W_{L}^{\prime}}$. 


\section{$5 W \gamma / Z \gamma$ Resonances}

Despite the search for diboson resonances playing a significant role in probing the source of EWSB, dynamical mechanisms of EWSB as well as mass generation of fermions, are still to be confirmed and may involve heavy bosons with spin 0 or 1 . An analysis was performed to search for heavy resonances decaying to $W \gamma$ and $Z \gamma$ final states, where both the $W$ and $Z$ bosons decay leptonically. Such decay modes from heavy resonances can emerge from BSM models with new scalar $[48,49]$ and vector [50] particles. The Low Scale Technicolour (LSTC) model [51] as well as a phenomenological model $[52,53]$ describing a singlet scalar particle $\phi$ decaying to $Z \gamma$ are used as benchmark models for this search. The LSTC model includes four technimesons, namely $a_{T}, \omega_{T}, \rho_{T}$ and $\pi_{T}$, with the following assumptions on the relationship between their masses: $m_{a_{T}} \approx m_{\rho_{T}}, m_{\rho_{T}}=m_{\omega_{T}}$ and $m_{\rho_{T}}-m_{\pi_{T}}=m_{W}$. These assumptions along with their decay modes $\left(\rho_{T} \rightarrow W Z, W \gamma, \omega_{T} \rightarrow Z \gamma\right.$ and $\left.a_{T} \rightarrow W \gamma\right)$ enable the test of the model via only $m_{\omega_{T}}$ and $m_{a_{T}}$. The scalar particle $\phi$ with spin 0 is neutral and can be composite, and it can also act as the pseudo-Goldstone boson in dynamical EWSB. The decay mode to dibosons $\phi \rightarrow W W / Z Z$ is largely supressed due to the singlet nature of EWSB, while the model also forbids the SM Yukawa interaction between $\phi$ and the fermions, disallowing $t \bar{t}$ and $b \bar{b}$ final states. The analysis is divided into the electron and muon channels, and indepedently searches for resonances by reconstructing the mass spectrum of the four possible final states, $m_{e v \gamma}, m_{e e \gamma}, m_{\mu \nu \gamma}, m_{\mu \mu \gamma}$. Sinco no excess over SM expectations is observed, the results are interpreted as 95\% CL limits on $m_{\phi}, m_{\omega_{T}}$ and $m_{a_{T}}$.

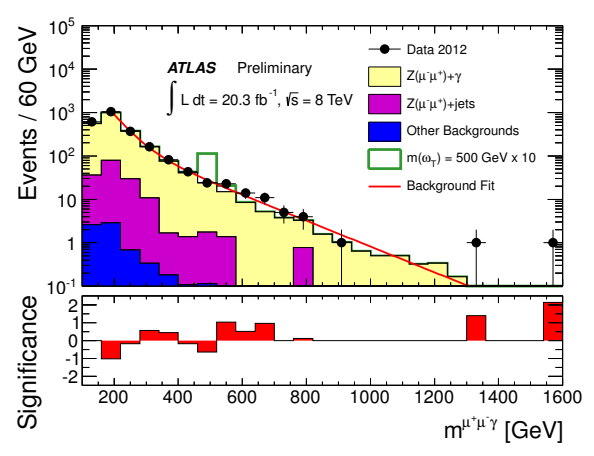

(a)

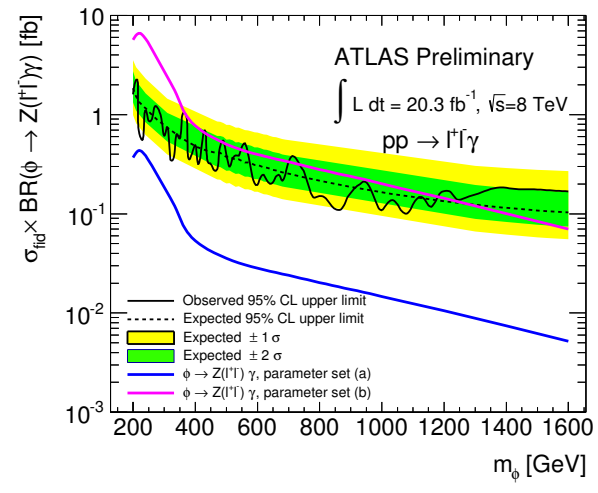

(b)

Figure 5: Shown in Figure 5a is the $m_{\mu \mu \gamma}$ distribution used to search for resonances, and in Figure 5b is the $95 \%$ CL limits on cross section times the branching ratio as a function of $m_{\phi}$. [54]

\section{Long Lived Neutral Particles}

Some BSM models involve a hidden sector that weakly couples to the SM via a heavy scalar particle $\Phi_{H S}$ [55-58], where a confining gauge interaction invisible to the SM is predicted. This communicator particle couples to mass in the same way as the Higgs boson. Current coupling measurements of the Higgs boson do not exclude exotic decays, and a $\Phi_{H S}$ with the mass of the Higgs boson is considered as a Higgs with an exotic decay channel. A Hidden Valley (HV) model [57, 58], where 
the lightest HV particles form an isospin triplet called Valley pions $\pi_{v}$, is used as a benchmark model. These valley pions would be produced in pairs $\left(\Phi \rightarrow \pi_{v} \pi_{v}\right)$ from the $p p$ collisions inside the ATLAS detector, with each of them decay further into a pair of fermions. Such decays into jets of the valley pions are predicted to be much later compared to the other processes produced from the $p p$ collisions. This analysis focuses on the decays located in the range from the outer edge of the Electromagnetic Calorimeter (ECAL) and into the Hadronic Calorimeter (HCAL). Figure 6a shows a simulated distribution of $\log _{10}\left(E_{H} / E_{E M}\right)$ versus the distance from the interaction point, where a jump in the energy deposited in this targeted region is clearly visible. The benchmark model predicts that approximately $20-30 \%$ of the producecd $\pi_{v}$ would decay in this targeted region. A special trigger is used to trigger those events as the emerging jets with displaced vertices, as such events are usually rejected by most other analyses. SM Multijet events are the main source of the background process, their contribution is estimated by using a multijet data sample in order to predict the probablility that a jet passes the trigger and analysis selection cuts. The observed data agree with the SM-only predictions and the results are interpreted as $95 \% \mathrm{CL}$ limits on the range of decay lengths of $\pi_{v}$ for several different combinations of $m_{\Phi}$ and $m_{\pi_{v}}$, for various values of the branching ratios.

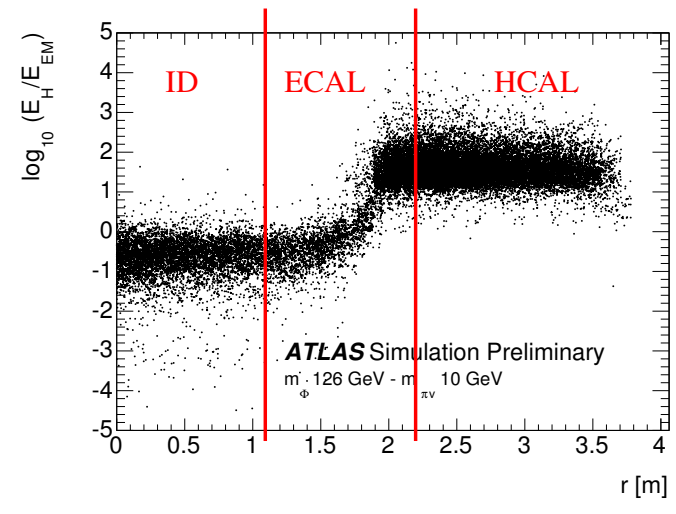

(a)

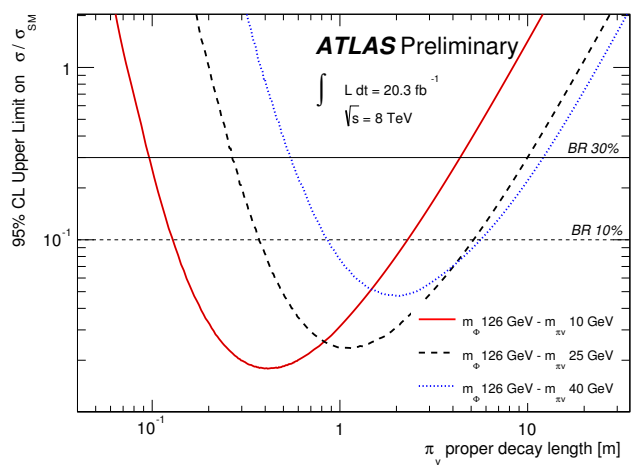

(b)

Figure 6: In Figure 6a is a simulated $\log _{10}\left(E_{H} / E_{E M}\right)$ distribution as a function the $\pi_{v}$ radial decay position, obtained from the generated MC signal sample with $m_{\phi}=126 \mathrm{GeV}$ and $m_{\pi_{v}}=10 \mathrm{GeV}$. Figure $6 \mathrm{~b}$ is the observed 95\% CL limits of several values of $m_{\pi_{v}}$ on $\sigma / \sigma_{S M}$ for $m_{\phi}=126 \mathrm{GeV}$ as a function of the proper decay length of $\pi_{v}$. [59]

\section{Contact Interaction and Large Extra Dimensions}

Dilepton final states resulting from new physics phenomena appear in many BSM theories, including resonances that have been searched by the ATLAS detector [60]. A search for non-resonant phenomena that appear as broad deviations from the SM have also been performed, with the analysis concentrated on investigating such phenomena arising from Contact Interactions (CI) and Large Extra Dimensions (LED).

The four fermion interaction that was formulated by Fermi [61] provides a non-renormalizable description for direct evidence of new gauge bosons from new interactions at much higher energies. 
Their presence can cause deviations from the SM in $p p$ collisions due to the compositness of quarks and leptons, where the binding energy of the fermion constituents are represented by a characteristic energy scale $\Lambda$. The cross section of the process $q \bar{q} \rightarrow l l$ in presence of contact interactions can be described as $\sigma_{t o t}=\sigma_{D Y}+\eta_{i j} \frac{F_{I}}{\Lambda^{2}}+\frac{F_{C}}{\Lambda^{4}}$, where $\sigma_{D Y}$ accounts for the SM Drell-Yan (DY) process $q \bar{q} \rightarrow Z / \gamma^{*} \rightarrow l l$, the second term describes the interference between the DY and CI process, while the third term represents the pure CI process. $F_{I}$ and $F_{C}$ are treated as functions of the cross-section and they do not depend on $\Lambda$.

A model that involves the existence of large flat extra dimensions, where gravity is allowed to propagate into and dilute its effects in the $3+1$ spatial dimensions, has been proposed by ArkaniHamed, Dimopoulos and Dvali (ADD) [62], which brings a solution to the vast hierachy between the Planck and EW scales. In this model, the string scale $M_{S}$ that represents the ultraviolet cutoff for the KK modes, can be used to describe the potential deviation from the SM in $p p$ collisions. These KK modes are responsible for the production of dileptons via virtual KK gravitions exchange (which is related to the fundamental Planck scale in $4+n$ dimensions). The cross section for the $q \bar{q} / g g \rightarrow l l$ process can be described (in a similar manner to the CI scenario) by $\sigma_{t o t}=\sigma_{D Y}+\mathscr{F} \frac{F_{i n t}}{M_{S}^{4}}+$ $\mathscr{F}^{2} \frac{F_{G}}{M_{S}^{8}}$. The functions of cross sections $F_{I}$ and $F_{G}$ involving respectively the interference and the pure KK effects, are considered to be independent of $M_{S}$. The interaction strength is characterised by $\mathscr{F} / M_{S} S^{4}$, and calculation for the dimensionless parameter $\mathscr{F}$ varies between models proposed by Giudice-Rattazzi-Wells (GRW) [63], Hewett [64] and Han-Lykken-Zhang (HLZ) [65].

The $m_{e e}$ and $m_{\mu \mu}$ spectrums were independently used to search for excess over SM expectations, the $\cos \theta^{*}$ distribution was also used to search for evidence of contact ineractions, where $\theta^{*}$ is the dilepton decay angle. As no significant excess was observed, the results from both channels are combined and interpreted as $95 \%$ CL limits on the values of the parameters $\Lambda$ and $M_{S}$ in the various considered models. Searches for signs of CI and ADD models of LED with different final states have also been performed with the ATLAS detector and reported in [22, 66-68].

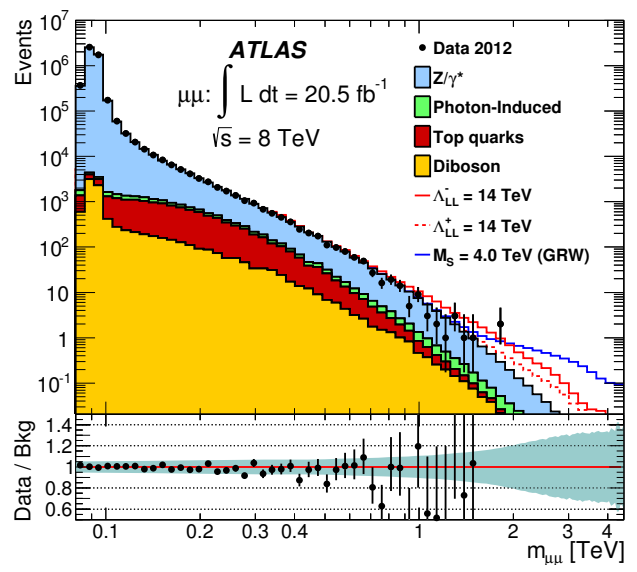

(a)

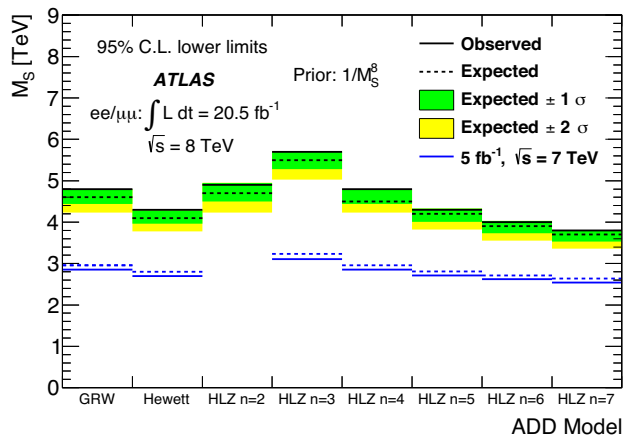

(b)

Figure 7: Shown in Figure 7a is the $m_{\mu \mu}$ distribution used to search for excess in the muon channel, and in Figure $7 \mathrm{~b}$ is the $95 \%$ CL limits on the value of the $M_{S}$ parameter obtained for the several considered models. [69] 


\section{Heavy Quarks}

The violation of "naturalness", when extrapolated above the EW energy scale, remains one of the open questions of the SM. The quadratic mass-squared divergences of fundamental scalar fields requires "fine tuning" to be compensated by some mechanism. This issue is commonly addressed in BSM theories by proposing a new symmetry, where the new states of the SM bosons and fermions related to such symmetry introduces new interactions that cancel out the divergency. These new states are generically strongly coupled resonances of some new confining dynamics, which can appear as vectorlike quarks (VLQ). Several non-SUSY BSM theories include vector-like quarks as a characteristic feature [70]. A search for the production of charge $+2 / 3(T)$ and $-1 / 3(B)$ VLQ that decays to a $Z$ boson and a third generation quark $(T \rightarrow Z t$ and $B \rightarrow Z b)$ has been performed. They can either be pair produced from the $p p$ collisions via the strong interaction, or singly produced via the EW interaction. The event selection require at least a pair of leptons to reconstruct a $Z$ boson candidate, and the selected candidate events are then divided into dilepton and trilepton events, depending on the presence of a third lepton that is not associated with the $Z$ candidate. The dilepton channel uses the $m_{Z}$ variable to search for excess over SM expectations, while the trilepton channel uses the transverse momentum of all central jets in the event $\left(H_{T}\right)$. Both of these channels are split further into two categories depending on the number of $b$-jets in the event: $N_{b t a g}=1, \geq 2$ for the dilepton channel, and $N_{\text {btag }}=0, \geq 1$ for the trilepton channel. The dilepton channel targets the pair production hypotheses, while the trilepton channel also accounts for single production hypotheses. As no excess over the SM predictions have been observed, the results from both dilepton and trilepton channels are combined and interpreted as $95 \%$ CL limits on the cross section for both the single and pair production, as a function of the mass of both $T$ and $B$ quarks. Limits are also derived for all heavy quark branching ratios that are consistent with the three possible decay modes ( $Z, W$ or $H$ bosons) by combining the results from both the dilepton and trilepton channels.

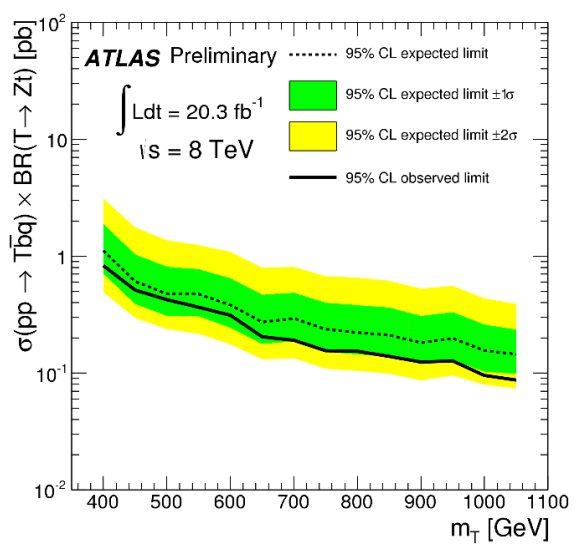

(a)

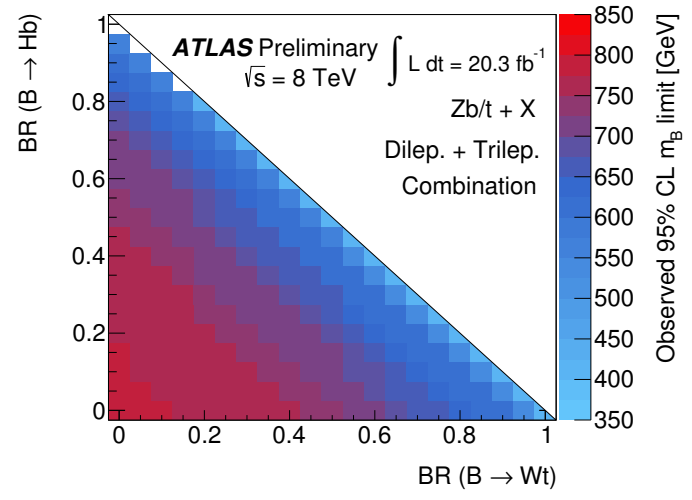

(b)

Figure 8: Shown in Figure 8a is the 95\% CL limits on the cross section times the branching ratio of the $Z$ decay mode as a function of mass for the single production of the $T$ quark, while Figure $8 \mathrm{~b}$ is the $95 \%$ CL limit of the pair production of the $B$ quark, presented in the branching ratio plane of $(W t, H b)$. [71] 


\section{Conclusion}

There have been many great achievements with the ATLAS detector during Run I of the LHC, such as the discovery of the Higgs boson. However there have yet been any observations of new physics, and many of the open questions of the SM still remain to be answered. With Run II starting in early 2015 with $\sqrt{s}=13 \mathrm{TeV}$, the search for BSM physics in both the Exotics and SUSY sectors, as well as precision measurements on the SM including the properties of the Higgs boson, will continue with exciting prospects.

\section{Acknowledgements}

I would like to thank the Federal Ministry of Education and Research of Germany (Bundesministerium für Bildung und Forschung) for providing the funding to deliver this report. I would also like to extend my gratitude to the conferene organizers for arranging such a pleasant and warm environment for exchanging scientific ideas, as well as to the participants for the fruitful conversations during the course of the conference.

\section{References}

[1] L. Evans and P. Bryant (editors) 2008 JINST 3 S08001

[2] ATLAS Collaboration, 2008 JINST 3 S08003

[3] ATLAS Collaboration, Phys. Lett. B 716 (2012) 1-29

[4] ATLAS Collaboration, ATLAS-CONF-2013-034, http://cds.cern.ch/record/1528170.

[5] ATLAS Collaboration, ATLAS-CONF-2013-040, http://cds.cern.ch/record/1542341.

[6] E. Eichten and K. Lane, Phys. Lett. B 669, 235-238 (2008).

[7] S. Catterall, L. Del Debbio, J. Giedt, and L. Keegan, Phys. Rev. D 85, 094501 (2012).

[8] J. Andersen et al., Eur. Phys. J. Plus 126, 81 (2011).

[9] L. Randall and R. Sundrum, Phys. Rev. Lett. 83, 3370 (1999).

[10] L. Randall and R. Sundrum, Phys. Rev. Lett. 83, 4690 (1999).

[11] H. Davoudiasl, J. L. Hewett and T. G. Rizzo, Phys. Rev. D 63, 075004 (2001).

[12] G. Altarelli, B. Mele, and M. Ruiz-Altaba, Z. Phys. C 45, 109 (1989).

[13] K. Agashe et al., Phys. Rev. D 76, 036006 (2007).

[14] G. Altarelli, B. Mele, and M. Ruiz-Altaba, Z. Phys. C 47, 676 (1990).

[15] M. Cacciari, G. P. Salam, and G. Soyez, J. High Energy Phys. 0804 (2008) 063.

[16] M. Cacciari and G. P. Salam, Phys. Lett. B 64157 (2006).

[17] Yu. L. Dokshitzer, G. D. Leder, S. Moretti, and B. R. Webber, J. High Energy Phys. 9708 (1997) 001.

[18] M. Wobisch and T. Wengler, arXiv:hep-ph/9907280.

[19] ATLAS Collaboration, ATLAS-CONF-2014-039, http://cds.cern.ch/record/1735253.

[20] D. Pappadopulo, A. Thamm, R. Torre, A. Wulzer, Heavy Vector Triplets: Bridging Theory and Data, arXiv:1402.4431.

[21] ATLAS Collaboration, CERN-PH-EP-2014-094, arXiv:1406.4456

[22] ATLAS Collaboration, CERN-PH-EP-2014-147, arXiv:1401.1376

[23] M. Chizhov, Phys. Part. Nucl. Lett. 8, 512 (2011), arXiv:1005.4287 [hep-ph].

[24] M. Chizhov and G. Dvali, Phys. Lett. B 703, 593 (2011). 
[25] M. Chizhov, V. Bednyakov, and J. Budagov, Phys. Atom. Nucl. 75, 90 (2012).

[26] M. Chizhov, V. Bednyakov, and J. Budagov, (2011), arXiv:1106.4161 [hep-ph].

[27] L. A. Anchordoqui, J. L. Feng, H. Goldberg, and A. D. Shapere, Phys. Lett. B 594, 363 (2004), arXiv:hep-ph/0311365 [hep-ph].

[28] P. Meade and L. Randall, J. High Energy Phys. 0805, 003 (2008), arXiv:0708.3017 [hep-ph].

[29] X. Calmet, W. Gong, and S. D. Hsu, Phys. Lett. B 668, 20 (2008), arXiv:0806.4605 [hep-ph].

[30] D. M. Gingrich, J. Phys. G 37, 105008 (2010), arXiv:0912.0826 [hep-ph].

[31] U. Baur, I. Hinchliffe, and D. Zeppenfeld, Int. J. Mod.Phys. A 2, 1285 (1987).

[32] U. Baur, M. Spira, and P. M. Zerwas, Phys. Rev. D 42,815 (1990).

[33] P. H. Frampton and S. L. Glashow, Phys. Lett. B , 157 (1987).

[34] P. H. Frampton and S. L. Glashow, Phys. Rev. Lett. 58, 2168 (1987).

[35] J. Bagger, C. Schmidt, and S. King, Phys. Rev. D 37, 1188 (1988).

[36] T. Han, I. Lewis, and Z. Liu, J. High Energy Phys. 12, 085 (2010), arXiv:1010.4309 [hep-ph].

[37] H. Georgi, E. E. Jenkins, and E. H. Simmons, Nucl. Phys. B 331, 541 (1990).

[38] C. Grojean, E. Salvioni, and R. Torre, J. High Energy Phys. 1107, 002 (2011), arXiv:1103.2761 [hep-ph].

[39] M. Cvetic and J. C. Pati, Phys. Lett. B 135, 57 (1984).

[40] Y. Mimura and S. Nandi, Phys. Lett. B 538, 406 (2002), arXiv:hep-ph/0203126 [hep-ph].

[41] CMS Collaboration, Phys. Lett. B 701, 160 (2011), arXiv:1103.0030 [hep-ex].

[42] ATLAS Collaboration, Phys. Lett. B 705, 28 (2011), arXiv:1108.1316 [hep-ex].

[43] E. Malkawi, T. M. Tait, and C.-P. Yuan Phys. Lett. B385 (1996) 304-310, arXiv:hep$\mathrm{ph} / 9603349$.

[44] M. Perelstein Prog. Part. Nucl. Phys. 58 (2007) 247-291, arXiv:hep-ph/0512128.

[45] G. Burdman, B. A. Dobrescu, and E. Pontón Phys. Rev. D74 (2006) 075008, arXiv:hepph/0601186.

[46] Z. Sullivan Phys. Rev. D66 (2002) 075011, arXiv:hep-ph/0207290.

[47] ATLAS Collaboration, CERN-PH-EP-2014-152, arXiv:1408.0886

[48] Benjamin C. Allanach, Jordan P. Skittrall and K. Sridhar, JHEP 0711, (2007) 089.

[49] A. Freitas and P. Schwaller, JHEP 1101, (2011) 022.

[50] K. D. Lane and E. Eichten, Phys. Lett. B 222 (1989) 274.

[51] K. D. Lane and E. Eichten, Phys. Lett. B 669, (2008) 235

[52] Ian Low, Joseph Lykken and Gabe Shaughnessy, Phys. Rev. D 84 (2011) 035027.

[53] R. Barbieri, R. Torre, Phys. Lett. B 695 (2011) 259.

[54] ATLAS Collaboration, CERN-PH-EP-2014-159, arXiv:1407.8150

[55] S. Chang, P.J. Fox and N. Weiner JHEP 0608 (2006) 068. arXiv:0511250 [hep-ph].

[56] S. Chang, R. Dermisek, J. F. Gunion and N. Weiner, Ann. Rev. Nucl. Part. Sci. 58, (2008) 75-98.

[57] M. J. Strassler and K. M. Zurek, Phys. Lett. B 651, (2007) 374-379, arXiv:0604261 [hep-ph].

[58] M. J. Strassler and K. M. Zurek, Phys. Lett. B 661, (2008) 263-267, arXiv:0605193 [hep-ph].

[59] ATLAS Collaboration, ATLAS-CONF-2014-041, http://cds.cern.ch/record/1740972.

[60] ATLAS Collaboration, CERN-PH-EP-2014-053, arXiv:1405.4123

[61] E. Fermi, Z. Phys. 88, 161 (1934)

[62] N. Arkani-Hamed, S. Dimopoulos, G. Dvali, Phys. Lett. B 429, 263 (1998), arXiv:hep$\mathrm{ph} / 9803315$.

[63] G.F. Giudice, R. Rattazzi, J.D. Wells, Nucl. Phys. B 544, 3 (1999), arXiv:hep-ph/9811291. 
[64] J.L. Hewett, Phys. Rev. Lett. 82, 4765 (1999), arXiv:hep-ph/9811356

[65] T. Han, J.D. Lykken, R.J. Zhang, Phys. Rev. D 59, 105006 (1999), arXiv:hep-ph/9811350.

[66] ATLAS Collaboration, ATLAS-CONF-2013-051, http://cds.cern.ch/record/1547567.

[67] ATLAS Collaboration, J. High Energy Phys. 08, 103 (2014).

[68] ATLAS Collaboration, Phys. Rev. Lett 112, 091804 (2014).

[69] ATLAS Collaboration, CERN-PH-EP-2014-135, arXiv:1407.2410

[70] J. Berger, J. Hubisz, and M. Perelstein, J. High Energy Phys. 07016 (2012).

[71] ATLAS Collaboration, ATLAS-CONF-2014-036, http://cds.cern.ch/record/1735195 